\title{
Evolutionary patterns of range size, abundance and species richness in Amazonian angiosperm trees
}

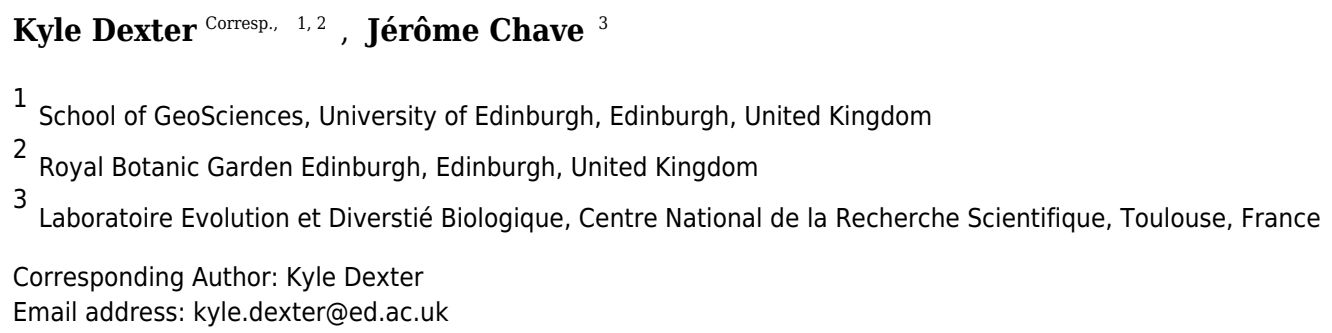

Amazonian tree species vary enormously in their total abundance and range size, while Amazonian tree genera vary greatly in species richness. The drivers of this variation are not well understood. Here, we construct a phylogenetic hypothesis that represents half of Amazonian tree genera in order to contribute to explaining the variation. We find several clear, broad-scale patterns. Firstly, there is significant phylogenetic signal for all three characteristics; closely related genera tend to have similar numbers of species and similar mean range size and abundance. Additionally, the species richness of genera shows a significant, negative relationship with the mean range size and abundance of their constituent species. Our results suggest that phylogenetically correlated intrinsic factors, namely traits of the genera themselves, shape among lineage variation in range size, abundance and species richness. We postulate that tree stature may be one particularly relevant trait. However, other traits may also be relevant, and our study reinforces the need for ambitious compilations of trait data for Amazonian trees. In the meantime, our study shows how large-scale phylogenies can help to elucidate, and contribute to explaining, macroecological and macroevolutionary patterns in hyperdiverse, yet poorly understood regions like the Amazon Basin. 
1 Evolutionary patterns of range size, abundance and species richness in Amazonian

2 angiosperm trees

3 Kyle G. Dexter ${ }^{1,2}$ and Jérôme Chave ${ }^{3}$

$4 \quad{ }^{1}$ School of Geosciences, University of Edinburgh, Edinburgh, U.K.

$5 \quad{ }^{2}$ Royal Botanic Garden Edinburgh, Edinburgh, U.K.

$6 \quad{ }^{3}$ Laboratoire de Evolution et Diversité Biologique, Centre National de la Recherche

7 Scientifique/Université Paul Sabatier, UMR 5174, Toulouse, France

8

9 Corresponding author:

10 Kyle G. Dexter

11 School of Geosciences, University of Edinburgh

12 201 Crew Building, King's Buildings,

13 Edinburgh EH9 3FF, United Kingdom

14 Phone: +44(0) 1316517065

15 Email: kyle.dexter@ed.ac.uk

17 Abstract word count: 195

18 Main text word count (excluding refs and legends): 3467

19 Number of Tables: 1

20 Number of Figures: 2

21 Number of References: 34

23 Running Header: Evolutionary Patterns of Amazonian Trees 


\section{ABSTRACT}

25 Amazonian tree species vary enormously in their total abundance and range size, while

26 Amazonian tree genera vary greatly in species richness. The drivers of this variation are not well

27 understood. Here, we construct a phylogenetic hypothesis that represents half of Amazonian tree

28 genera in order to contribute to explaining the variation. We find several clear, broad-scale

29 patterns. Firstly, there is significant phylogenetic signal for all three characteristics; closely

30 related genera tend to have similar numbers of species and similar mean range size and

31 abundance. Additionally, the species richness of genera shows a significant, negative relationship

32 with the mean range size and abundance of their constituent species. Our results suggest that

33 phylogenetically correlated intrinsic factors, namely traits of the genera themselves, shape

34 among lineage variation in range size, abundance and species richness. We postulate that tree

35 stature may be one particularly relevant trait. However, other traits may also be relevant, and our

36 study reinforces the need for ambitious compilations of trait data for Amazonian trees. In the

37 meantime, our study shows how large-scale phylogenies can help to elucidate, and contribute to

38 explaining, macroecological and macroevolutionary patterns in hyperdiverse, yet poorly

39 understood regions like the Amazon Basin. 


\section{Introduction}

48 Some Amazonian tree species attain incredibly high abundance (tens to hundreds of millions of

49 mature individuals), while most have small populations sizes, numbering in the thousands to tens

50 of thousands of individuals (ter Steege et al. 2013, 2015). Similarly, the range of some

51 Amazonian tree species extends across the entire Amazon basin, while most are restricted to

52 much smaller areas (Kristiansen et al. 2009). A similar imbalance is observed in species to genus

53 ratios. Over half of all Amazonian tree species belong to genera with 100 or more species, while

54 the majority of genera (52\%) have ten or fewer species (Gentry 1993). The drivers of variation in

55 these fundamental characteristics of tree species and genera are poorly known, despite the

56 Amazon representing the largest tropical forest in the world and housing the greatest number of

57 tree species of any forest (Fine \& Ree 2006).

59 Variation in the abundance of tree species at the community level is evident in any forest and has

60 been studied in tropical forests since at least 1979 (Hubbell 1979). However, only relatively

61 recently has sufficient taxonomic and forest inventory work been carried out at sufficiently large

62 spatial scales $(>1000 \mathrm{~km})$ to explore patterns of dominance across the Amazon; the results have

63 shown conclusively that certain tree species dominate at large spatial scales as well (Pitman et al.

64 2001, ter Steege et al. 2013, 2015). Neutral ecological models where species drift randomly in

65 abundance over time can generate variation in abundance, but they fail to explain the consistent

66 dominance of some tree species across the Amazon (Hubbell 2001, Condit et al. 2002). This

67 begs the question of which characteristics or traits allow certain tree species to dominate, but the

68 absence of adequate trait datasets has limited attempts to answer this question. There are two

69 large-scale databases available that provide reasonable coverage of Amazonian tree species, for 
70 wood density and seed mass (Zanne et al. 2009, Royal Botanic Gardens Kew 2016), but these

71 two traits do not appear to be related to tree abundance in the Amazon (ter Steege et al. 2013,

72 2015). At smaller spatial scales, tree height has been shown to be positively correlated with

73 abundance (Pitman et al. 2001, Arellano et al. 2014), but it is unclear if this pattern holds at the

74 scale of the entire Amazon Basin.

75

76 There has been less progress in studying variation in the range size of Amazonian trees, in large

77 part because many areas of the Amazon remain poorly known by botanical scientists. In 1999,

78 Pitman and colleagues noted that "not a single tree species distribution in the Amazon basin has

79 been reliably mapped" (Pitman et al. 1999), and this remains the case. Nevertheless, with some

80 simplifying assumptions, Feeley and Silman (2009) succeeded in providing a first estimate of the

81 range size of thousands of Amazonian plant species. These authors documented substantial

82 variation in the range size of Amazonian tree species, but, given their focus on conservation, they

83 did not attempt to explain this variation, and neither has any subsequent study. Range size has

84 been shown to be positively related to the total abundance of tree species in the Amazon (ter

85 Steege et al. 2013), but the arrow of causality probably goes in the reverse direction (i.e. tree

86 species with larger ranges can achieve greater total abundance). Meanwhile, studies of a more

87 limited taxonomic scope have shown that range size in Amazonian palm tree species (Arecaceae)

88 is positively related to their height (Ruokolainen et al. 2002; Kristiansen et al. 2009). Whether

89 this pattern holds for dicotyledenous Amazonian trees is unknown, and whether other tree traits

90 are related to range size remains to be explored. 
92 Lastly, while variation in the species richness of tree genera in the Amazon has been noted (e.g.

93 Bermingham \& Dick 2001), it has received surprisingly little research attention compared to the

94 extensive efforts directed toward understanding the extraordinary total tree species richness of

95 the Amazon (Prance 1982, Hoorn et al. 2010). Baker et al. (2014) represents a landmark in this

96 regard, as the authors focused on explaining variation in species richness amongst 51 Amazonian

97 tree lineages (primarily genera). The authors focused on assessing the role of intrinsic factors

98 (i.e. traits of the lineages themselves) as compared to extrinsic factors (e.g. geological events) in

99 explaining this variation. They showed that the species richness of genera was negatively related

100 with their turnover time; genera that showed higher mortality and recruitment rates in forest

101 inventory plots also had more species. Meanwhile, they did not find a relationship of species

102 richness with dispersal syndrome, breeding system or tree height. The negative results in this

103 study may be best considered as preliminary however, given that the study covered only 51 tree

104 genera (among 100s in the Amazon) and only trees $\geq 10 \mathrm{~cm}$ diameter at breast height $(1.3 \mathrm{~m}$

105 above the ground), which was the minimum size threshold for sampling trees in the forest

106 inventory plots used to estimate turnover times.

107

108 Acquiring sufficient trait data to thoroughly evaluate the drivers of variation in the abundance,

109 range size and species richness of Amazonian tree lineages will require many costly field

110 campaigns that will likely take multiple decades to complete. In the meantime, alternative

111 approaches should be pursued. One fundamental question is whether intrinsic or extrinsic factors

112 are more important. To the degree that intrinsic factors or traits (e.g. tree height or dispersal

113 syndrome) show phylogenetic signal (cf. Freckleton et al. 2002, Blomberg et al. 2003), large-

114 scale phylogenies could be used to test the role of intrinsic factors in driving variation in 
115 abundance, range size and species richness. In other words, if closely related lineages tend to

116 have similar abundance, range size and species richness, then phylogenetically correlated traits

117 are likely to be important drivers of variation in these characteristics. It is improbable that

118 extrinsic factors would generate phylogenetic signal for abundance, range size or species

119 richness within a given biogeographic region. Recent advances in the generation of sequence

120 data for large numbers of Amazonian tree genera (e.g. Baraloto et al. 2012) mean that

121 phylogenetic approaches are now feasible. Here, we generate a temporally-calibrated

122 phylogenetic hypothesis that includes half of all Amazonian tree genera. We use this genus-level

123 phylogeny to assess if there is phylogenetic signal for the mean range size, mean abundance and

124 species richness of genera, with the aim of testing the importance of intrinsic traits of genera in

125 driving macroecological and macroevolutionary patterns in Amazonian trees.

126

127 Methods

128 We intersected a list of all Neotropical tree genera (from

129 http://ctfs.si.edu/webatlas/neotropicaltree/) with a list of Amazonian plant species (Feeley \&

130 Silman 2009) in order to generate a list of Amazonian tree species. The Feeley and Silman

131 (2009) dataset additionally includes estimates of range size for all species. We obtained estimates

132 for the total abundance of Amazonian tree species from ter Steege et al. (2013).

133

134 We obtained sequences of the $r b c L$ plastid gene for 631 Amazonian angiosperm tree genera

135 (Table S1), with 567 sequences coming from Genbank (www.ncbi.nlm.nih.gov/genbank/) and an

136 additional 64 genera being newly sequenced following protocols outlined in Baraloto et al.

137 (2012). We obtained sequences of the matK plastid gene from Genbank for 452 of the 631 
138 genera with $r b c L$ data (Table S1). Sequences were aligned using the MAFFT software (Katoh \&

139 Standley 2013). 'Ragged ends' of the sequences that were missing data for most genera were

140 manually deleted from the alignment. Preliminary phylogenetic analyses allowed us to exclude

141 sequences from GenBank for genera that were phylogenetically placed in a different family to

142 that which they are thought to belong taxonomically. The final alignment can be found in the

143 Supplementary Material.

145 We estimated a maximum likelihood phylogeny for the genera in RAxML v8.0.0 (Stamatakis,

146 Hoover \& Rougemont 2008), on the CIPRES web server (www.phylo.org). We used the default

147 settings, including a General Time Reversible (GTR) + Gamma (G) model of sequence

148 evolution, with separate models for the $r b c L$ and matK genes (i.e. a partitioned analysis). We

149 included sequences of Amborella trichopoda (Amborellaceae) and Nymphaea alba

150 (Nymphaeaceae) as outgroups. This phylogeny (see Supplementary Material) was used as a

151 starting tree for simultaneous topology and divergence time estimation in the software BEAST

152 v1.82 (Drummond \& Rambaut 2007). We implemented fossil-based age constraints for 25 nodes

153 distributed across the phylogeny, using log-normal prior distributions with an offset to impose a

154 hard minimum age (see Table S2). We used a GTR + G model of sequence evolution, with

155 separate models for the $r b c L$ and matK genes, an uncorrelated relaxed lognormal clock, and a

156 birth-death model for the speciation process. We conducted several preliminary runs to optimise

157 the tuning and weight of parameters as per recommendations generated by the software. Once

158 parameter optimisation stabilised, we ran two separate chains for 100 million generations. The

159 first 50 million generations of each chain were discarded as "burn-in", as the posterior

160 probability of the phylogeny did not stabilise until this point. We combined the post burn-in 
161 posterior distributions of parameters and confirmed that effective sample size (ESS) values

162 exceeded 100 for all parameters. We then used the phyutility software (Smith \& Dunn 2008) to

163 generate an all-compatible consensus tree from the combined post burn-in posterior distribution

164 of trees. Node ages were optimised onto this consensus phylogeny as the median value for a

165 given node across all trees in the posterior distribution that contained the node (using the

166 TreeAnnotator software, Drummond \& Rambaut 2007).

167

168 For each genus in the phylogeny, we calculated the mean range size and abundance for all

169 constituent species in the Feeley and Silman (2009) and ter Steege et al. (2013) datasets. Of the

170631 genera in the phylogeny, 493 had an abundance estimate for at least one species in ter Steege

171 et al. (2013). We considered the number of species for each genus in the Feeley and Silman

172 (2009) dataset as an estimate of the species richness of that genus in the Amazon. As an

173 alternative estimate, we used the Neotropical species richness estimates for genera in Gentry

174 (1993), which produced highly similar results. We assessed correlations amongst these genus-

175 level characteristics using Pearson's correlation coefficients for both the raw values and for their

176 phylogenetically independent contrasts.

177

178 We tested for phylogenetic signal for each of these genus-level characteristics using Pagel's $\lambda$

179 (Freckleton, Harvey \& Pagel 2002). Under Brownian motion evolution, where trait values drift

180 randomly over evolutionary time and where the phylogenetic relationships of taxa perfectly

181 predict the covariance among taxa for trait values, the expected value of $\lambda$ is one. When the

182 phylogenetic relationships of taxa do not predict the covariance at all, the expected value of $\lambda$ is 
183 zero. We compared the fit of different values for $\lambda$ (one, zero and the maximum likelihood

184 estimate) using the Akaike information criterion (AIC).

185

186 In order to determine which lineages may be responsible for significant phylogenetic signal for a 187 given characteristic (e.g. mean range size of genera), we used the following approach. We first

188 estimated the ancestral value at each node in the phylogeny using maximum likelihood ancestral

189 state reconstruction (Schluter et al. 1997). We then randomised the tips of the phylogeny 1000

190 times, reconstructed ancestral values at nodes each time, and compared the observed

191 reconstructed value to that across the randomisations. If the observed value for a node was

192 greater than that in $97.5 \%$ of the randomisations, we considered the lineage descending from that

193 node to show significantly high values for the trait, while if the observed value was lower than

$1942.5 \%$ of the randomisations, we considered the lineage to show significantly low values.

195

196 In order to assess whether major clades (Magnoliids, Monocots, Rosids and Asterids) differ in

197 the species richness and mean range size and abundance of their constituent genera, we used

198 analyses of variance with major clade as the grouping variable. In order to determine which

199 clades may be driving significant results, we used Tukey’s tests. All analyses were conducted,

200 and figures constructed, in the R Statistical Software (R Core Development Team 2016) using

201 functions in the ape (Paradis et al. 2016), geiger (Harmon et al. 2016) and phytools (Revell

202 2016) packages (see Supplementary Material for codes).

203

204 Results 
205 The phylogeny derived from the DNA sequence dataset that we compiled spans from the

206 Magnoliids to the Asterids, thus encompassing all major clades of angiosperms (Fig. 1; see

207 Supplementary Material for newick-formatted tree file). The $64 \mathrm{rbcL}$ sequences that were

208 generated as part of this study represent $\sim 10 \%$ of the genera in the phylogeny, and they therefore

209 represent a valuable contribution to our understanding of the evolutionary relationships of

210 Amazonian tree genera. Most orders and families were monophyletic in the phylogeny with the

211 notable (previously known) exceptions of Olacaceae and Icacinaceae, while the large-scale

212 phylogenetic relationships are largely in agreement with those from recent, angiosperm-wide

213 phylogenetic analyses (e.g. Magallón et al. 2015).

215 The species richness of genera is negatively correlated with mean range size $(\mathrm{r}=-0.40, \mathrm{p}<$

$2160.001)$ and mean abundance $(r=-0.38, \mathrm{p}<0.001)$. These relationships are weaker, but still

217 significant, when using phylogenetically independent contrasts (PICs), indicating that

218 phylogenetically related traits partially underlie the correlations (mean range size PICs: $r=-0.28$,

$219 \mathrm{p}<0.001$; mean abundance PICs: $\mathrm{r}=-0.24, \mathrm{p}<0.001$; Fig. 2). Meanwhile, mean range size and

220 abundance of genera are strongly positively correlated, using both the raw data and PICs ( $\mathrm{r}=$

$2210.44, \mathrm{p}<0.001$; PICs: $\mathrm{r}=0.43, \mathrm{p}<0.001)$. All of the genus-level characteristics show significant

222 phylogenetic signal, but less than would be expected under a Brownian motion model of

223 evolution (Table 1).

225 Significant phylogenetic signal for these characteristics is driven by significantly high or low

226 values in many lineages (Fig. 1, Table S3). Diverse lineages in the Magnoliids and the Asterids

227 show high species richness and low mean range size and abundance, including the Lamiales and 
228 multiple lineages in the Rubiaceae and Solanales. One marked exception to the general pattern in

229 the Asterids is Lecythidaceae, which shows low species richness and high abundance.

230 Meanwhile, many lineages in the Rosids show low species richness and high mean range size

231 and abundance, including Euphorbiaceae, Salicaceae and Moraceae. Within the Rosids, the

232 exception to this pattern is found in multiple lineages in the Myrtales, including the

233 Melastomataceae, which show a pattern similar to most lineages in the Asterids. The

234 Leguminosae (Fabaceae), the most genus-rich family in our dataset, does not show any

235 significant departures from null expectations, although individual lineages therein show low

236 species richness and high mean range size. Within the monocots, the Arecaceae show low mean

237 range size, while one lineage (Iriartea with Socratea) shows particularly high abundance.

239 A non-phylogenetic comparison of genera in the major clades shows they are not significantly

240 different in terms of mean species richness (ANOVA for species richness: $F=1.18, p=0.317$ ),

241 while they are significantly different for mean range size and abundance, but the effect sizes are

242 small (range size: $F=10.56, p<0.001, R^{2}=0.05$; abundance: $F=7.13, p<0.001, R^{2}=0.04$ ).

243 Tukey's tests reveal that genera in the Rosids have significantly larger mean ranges, on average,

244 than genera in the Asterids $(\mathrm{p}<0.001)$ and Monocots $(\mathrm{p}=0.019)$, while genera in the Rosids

245 and Monocots have significantly higher mean abundance than genera in the Asterids $(p<0.001$

$246 \& \mathrm{p}=0.020$, respectively).

248 Discussion

249 Fundamental characteristics of Amazonian tree genera, namely their species richness and the

250 mean range size and abundance of their constituent species, show marked and significant 
251 phylogenetic signal (Table 1). In other words, closely related genera tend to have similar

252 numbers of species and species with similar range sizes and abundances (Fig. 1). These genus-

253 level characteristics are also strongly correlated with each other (Fig. 2). Our results suggest that

254 intrinsic factors (i.e. traits of the genera themselves) exert a strong influence on the range size,

255 abundance and species richness of Amazonian tree species and genera.

256

257 We propose that tree height may be one of the key traits underlying the observed results. Many

258 of the lineages in our study that show high species richness and small geographic ranges (e.g.

259 Myrtaceae, Melastomataceae, Rubiaceae, Asterales, Solanales, and Lamiales) tend to be small in

260 stature. Previous studies have shown a positive relationship between the height of Amazonian

261 trees and their range size (Ruokolainen et al. 2002, Kristiansen et al. 2009). Such a relationship

262 may be due to larger-statured trees being able to disperse their seeds greater distances, likely

263 through greater fecundity, which would increase the chances that at least some seeds make it a

264 long distance and would, for animal-dispersed species, potentially attract more dispersers.

265 Increased dispersal ability would also increase gene flow among distant populations, which, in

266 turn, could reduce opportunities for allopatric isolation and contribute to reduced diversification.

267 Smaller statured trees may also have shorter generation times, which could contribute to

268 increased diversification (Baker et al. 2014). Thus, small-stature may be a biological trait that

269 spurs diversification and may also underlie the negative correlation between mean range size and

270 species richness of genera. Small-statured lineages also show lower abundances in the datasets

271 assembled, although this is partly, if not entirely, explained by the abundance estimates being

272 derived from tree plots that survey individuals $>10 \mathrm{~cm}$ diameter at breast height (ter Steege et al.

273 2013). Meanwhile, the relationship between mean abundance and species richness of genera may 
274 simply be a by-product of correlations between range size and abundance. These are likely

275 necessarily related as the range size of a species will restrict the total abundance that it can

276 achieve.

277

278 Our focus on tree height as a key variable does not negate a role for other phylogenetically

279 correlated traits in contributing to the observed results. In local-scale studies of Amazonian tree

280 communities, most of the traits that have been examined (e.g. wood density, specific leaf area)

281 have shown significant phylogenetic signal (Kraft \& Ackerly 2010, Baraloto et al. 2012). Large-

282 scale compilations of trait data from across the Amazon are now needed in order to assess which

283 exact traits may be driving our results.

285 It is important to note that the phylogenetic signal we observe for these genus-level

286 characteristics is less than would be expected under a Brownian motion model of evolution. In

287 fact, the Brownian model fits the data much worse than a simple model whereby there is no

288 phylogenetic dependence to the observed values (Table 1). This result may be due to some

289 evolutionary process, such as selection, driving closely related genera to diverge in values for the

290 studied genus-level characteristics. However, in contrast to what might be thought for other traits

291 (e.g. those related to ecological niches such as resource acquisition or defence strategy), it is not

292 immediately clear why selection would favour divergence among closely related lineages in their

293 diversification rate, average range size or average abundance. Alternatively, a Brownian motion

294 model may be simply a poor descriptor of how these genus-level characteristics change over

295 time. The Brownian motion model stipulates that per unit time, small changes in trait values are

296 much more likely than large changes. It may be the case that large changes in range size, 
297 abundance and diversification rate among closely related changes are just as likely as small

298 changes. The exact pattern of change in these characteristics would depend on how the

299 underlying driving factors themselves, be they intrinsic or extrinsic, change over time and space.

300

301 In any case, the phylogenetic signal we document is evident across multiple phylogenetic scales.

302 At a broad scale, we find that various lineages in the Rosids are comprised of genera that show

303 low species richness and high mean range size and abundance, while lineages in the Magnoliids

304 and Asterids show the opposite pattern. Interestingly, a non-phylogenetic approach, using

305 analyses of variance, did not find large differences amongst these major lineages. This contrast in

306 results may be due in part to lineages within the major clades that belie the general pattern. For

307 example, the Myrtales (in the Rosids) show a pattern typical of the Asterids, while the

308 Lecythidaceae (in the Asterids) show a pattern typical of Rosids. The contrast is likely also due

309 to the manner in which these genus-level characteristics are distributed across the phylogeny.

310 While genera within a given major clade can show a diversity of values for these characteristics,

311 their relative phylogenetic positions result in reconstructed ancestral values that show systematic

312 differences amongst lineages in different major clades. A phylogenetic approach was thus

313 necessary to reveal how these major clades differ have differed over evolutionary time. This

314 phylogenetic approach has also served to show how phylogenetically-correlated factors, intrinsic

315 to lineages themselves, have contributed to the macroecological and macroevolutionary patterns

316 observed in present-day Amazonian trees.

317

318 Acknowledgements 
319 We wish to thank Julien Vieu, Mailyn Gonzalez, Darin Penneys, and Céline Vicédo for

320 generating DNA sequences, and Lourens Poorter, and Tim Baker for collecting new samples in

321 the field. We also thank two anonymous reviewers for helpful suggestions that improved the

322 manuscript.

323

324 References

325 Arellano, G., Loza, M. I., Tello, J. S. \& Macia, M. J. 2015. Commonness and rarity determinants

326 of woody plants in different types of tropical forests. Biodivers. Conserv. 24, 1073-1087.

327 (doi:10.1007/s10531-014-0843-y)

328 Baker T. R., R. T. Pennington, S. Magallon, E. Gloor, W. F. Laurance, M. Alexiades, E. Alvarez, 329 A. Araujo, E. J. M. M. Arets, G. Aymard, A. A. de Oliveira, I. Amaral, L. Arroyo, D. Bonal, 330 R. J. W. Brienen, J. Chave, K. G. Dexter, A. Di Fiore, E. Eler, T. R. Feldpausch, L. Ferreira, 331 G. Lopez-Gonzalez, G. van der Heijden, N. Higuchi, E. Honorio, I. Huamantupa, T. J.

332 Killeen, S. Laurance, C. Leaño, S. L. Lewis, Y. Malhi, B. S. Marimon, B. H. Marimon Junior, 333 A. Monteagudo Mendoza, D. Neill, M. C. Peñuela-Mora, N. Pitman, A. Prieto, C. A.

334 Quesada, F. Ramírez, H. Ramírez Angulo, A. Rudas, A. R. Ruschel, R. P. Salomão, A. S. de 335 Andrade, J. N. M. Silva, M. Silveira, M. F. Simon, W. Spironello, H. ter Steege, J. Terborgh, 336 M. Toledo, A. Torres-Lezama, R. Vasquez, I. C. G. Vieira, E. Vilanova, V. A. Vos, \& O. L. 337 Phillips. 2014. Fast demographic traits promote high diversification rates of Amazonian trees. 338 Ecol. Lett. 17, 527-36. (doi:10.1111/ele.12252)

339 Baraloto C., O. J. Hardy, C. E. T. Paine, K. G. Dexter, C. Cruaud, L. T. Dunning, M.-A.

340 Gonzalez, J.-F. Molino, D. Sabatier, V. Savolainen, \& J. Chave. 2012. Using functional traits 
341

342

343

344

345

346

347

348

349

350

351

352

353

354

355

356

357

358

359

360

361

362

363

and phylogenetic trees to examine the assembly of tropical tree communities. J. Ecol. 100, 690-701. (doi:10.1111/j.1365-2745.2012.01966.x)

Bermingham, E. \& Dick, C. 2001. The Inga - Newcomer or Museum Antiquity. Science 293, $2214-2216$.

Blomberg, S. P., Garland, T. \& Ives, A. R. 2003. Testing for phylogenetic signal in comparative data: Behavioral traits are more labile. Evolution 57, 717-745.

Condit, R., N. Pitman, E. G. Leigh, J. Chave, J. Terborgh, R. B. Foster, P. Nunez, S. Aguilar, R. Valencia, G. Villa, H. C. Muller-Landau, E. Losos, \& S. P. Hubbell. 2002, Beta-diversity in tropical forest trees. Science 295, 666-669.

Drummond, A. J. \& Rambaut, A. 2007. BEAST: Bayesian evolutionary analysis by sampling trees. BMC Evol. Biol. 7, 214. (doi:10.1186/1471-2148-7-214)

Feeley, K. J. \& Silman, M. R. 2009. Extinction risks of Amazonian plant species. Proc. Natl. Acad. Sci. U. S. A. 106, 12382-12387. (doi:10.1073/pnas.0900698106)

Fine, P. V. A. \& Ree, R. H. 2006. Latitudinal Gradient in Tree Diversity. Am. Nat. 168, 796-804. Freckleton, R. P., Harvey, P. H. \& Pagel, M. 2002. Phylogenetic analysis and comparative data: A test and review of evidence. Am. Nat. 160, 712-726. (doi: 10.1086/343873)

Gentry, A. H. 1993. A Field Guide to the Families and Genera of Woody Plants of Northwest South America. Washington D.C., U.S.A.: Conservation International.

Harmon, L., J. Weir, C. Brock, R. Glor, W. Challenger, G. Hunt, R. FitzJohn, M. Pennell, G. Slater, J. Brown, J. Uyeda, J. Eastman. 2016. Geiger R package: analysis of evolutionary diversification. http://lukejharmon.github.io/code/

Hoorn, C., F. P. Wesselingh, H. Steege, M. A. Bermudez, A. Mora, J. Sevink, I. Sanmartín, C. L. Anderson, J. P. Figueiredo, C. Jaramillo, D. Riff, F. R. Negri, H. Hooghiemstra, J. Lundberg, 
364 T. Stadler, T. Särkinen, \& A. Antonelli. 2010 Amazonia Through Time : Andean Uplift,

365 Climate Change, Landscape Evolution, and Biodiversity. Science 330, 927-931.

366 Hubbell, S. P. 1979. Tree dispersion, abundance, and diversity in a tropical dry forest. Science

$367 \quad 203,1299-1309$.

368 Hubbell, S. P. 2001. The Unified Neutral Theory of Biodiversity and Biogeography. Princeton

369 University Press, Princeton, NJ, USA.

370 Katoh, K. \& Standley, D. M. 2013. MAFFT multiple sequence alignment software version 7:

371 improvements in performance and usability. Mol. Biol. Evol. 30, 772-80.

$372 \quad$ (doi:10.1093/molbev/mst010)

373 Kraft, N. J. B. \& Ackerly, D. D. 2010. Functional trait and phylogenetic tests of community

374 assembly across spatial scales in an Amazonian forest. Ecol. Monogr. 80, 401-422.

$375 \quad$ (doi:10.1890/09-1672.1)

376 Kristiansen, T., Svenning, J.-C., Grandez, C., Salo, J. \& Balslev, H. 2009. Commonness of

377 Amazonian palm (Arecaceae) species: Cross-scale links and potential determinants. Acta

378 Oecologica-International J. Ecol. 35, 554-562. (doi:10.1016/j.actao.2009.05.001)

379 Magallón, S., Gómez-Acevedo, S., Sánchez-Reyes, L. L. \& Hernández-Hernández, T. 2015. A

380 metacalibrated time-tree documents the early rise of flowering plant phylogenetic diversity.

$381 \quad$ New Phytol. 207, 437-53. (doi:10.1111/nph.13264)

382 Paradis, E. 2016. Ape R package: analysis of phylogenetics and evolution. http://ape-

383 package.ird.fr/

384 Pitman, N. C. A., Terborgh, J., Silman, M. R. \& Nunez, P. 1999. Tree species distributions in an 385 upper Amazonian forest. Ecology 80, 2651-2661 . 
386 Pitman, N. C. A., Terborgh, J., Silman, M. R., Nunez, P., Neill, D. A., Ceron, C. E., Palacios, W. 387 A. \& Aulestia, M. 2001. Dominance and distribution of tree species in upper Amazonian terra 388 firme forests. Ecology 82, 2101-2117.

389 Prance, G. T. 1982. (Editor) Biological Diversification in the Tropics. Columbia Univ. Press, $390 \quad$ New York, NY, USA.

391 Purvis, A., Agapow, P. M., Gittleman, J. L. \& Mace, G. M. 2000. Nonrandom extinction and the 392 loss of evolutionary history. Science 288, 328-330. (doi:10.1126/science.288.5464.328)

393 R Core Development Team. 2016. R: A language and environment for statistical computing. R 394 Foundation for Statistical Computing, Vienna, Austria.

395 Royal Botanic Gardens Kew. 2016. Seed Information Database (SID). Version 7.1. Available 396 from: http://data.kew.org/sid/

397 Revell. 2016. Phytools R package: phylogenetic tools for comparative biology (and more).

398 http://www.phytools.org

399 Ruokolainen, K., Tuomisto, H., Vormisto, J. \& Pitman, N. 2002. Two biases in estimating range $400 \quad$ sizes of Amazonian plant species. J. Trop. Ecol. 18, 935-942.

$401 \quad$ (doi:10.1017/S0266467402002614)

402 Schluter, D., Price, T., Mooers, A. O. \& Ludwig, D. 1997. Likelihood of ancestor states in 403 adaptive radiation. Evolution. 51, 1699-1711. (doi:10.2307/2410994)

404 Smith, S. A. \& Dunn, C. W. 2008. Phyutility: a phyloinformatics tool for trees, alignments, and 405 molecular data. Bioinformatics. 24: 715-716 (doi: 10.1093/bioinformatics/btm619)

406 Stamatakis, A., Hoover, P. \& Rougemont, J. 2008 A Rapid Bootstrap Algorithm for the RAxML 407 Web Servers. Syst. Biol. 57, 758-771. (doi:10.1080/10635150802429642) 
408 ter Steege H., N. C. a Pitman, D. Sabatier, C. Baraloto, R. P. Salomão, J. E. Guevara, O. L.

409 Phillips, C. V Castilho, W. E. Magnusson, J.-F. Molino, A. Monteagudo, P. Núñez Vargas, J.

410 C. Montero, T. R. Feldpausch, E. N. H. Coronado, T. J. Killeen, B. Mostacedo, R. Vasquez,

411 R. L. Assis, J. Terborgh, F. Wittmann, A. Andrade, W. F. Laurance, S. G. W. Laurance, B. S.

412 Marimon, B.-H. Marimon, I. C. Guimarães Vieira, I. L. Amaral, R. Brienen, H. Castellanos,

413 D. Cárdenas López, J. F. Duivenvoorden, H. F. Mogollón, F. D. D. A. Matos, N. Dávila, R.

414 García-Villacorta, P. R. Stevenson Diaz, F. Costa, T. Emilio, C. Levis, J. Schietti, P. Souza,

415 A. Alonso, F. Dallmeier, A. J. D. Montoya, M. T. Fernandez Piedade, A. Araujo-Murakami,

416 L. Arroyo, R. Gribel, P. V. a Fine, C. a Peres, M. Toledo, G. a Aymard C, T. R. Baker, C.

417 Cerón, J. Engel, T. W. Henkel, P. Maas, P. Petronelli, J. Stropp, C. E. Zartman, D. Daly, D.

418 Neill, M. Silveira, M. R. Paredes, J. Chave, D. D. A. Lima Filho, P. M. Jørgensen, A. Fuentes, 419 J. Schöngart, F. Cornejo Valverde, A. Di Fiore, E. M. Jimenez, M. C. Peñuela Mora, J. F.

420 Phillips, G. Rivas, T. R. van Andel, P. von Hildebrand, B. Hoffman, E. L. Zent, Y. Malhi, A.

421 Prieto, A. Rudas, A. R. Ruschell, N. Silva, V. Vos, S. Zent, A. a Oliveira, A. C. Schutz, T.

422 Gonzales, M. Trindade Nascimento, H. Ramirez-Angulo, R. Sierra, M. Tirado, M. N. Umaña

423 Medina, G. van der Heijden, C. I. a Vela, E. Vilanova Torre, C. Vriesendorp, O. Wang, K. R.

424 Young, C. Baider, H. Balslev, C. Ferreira, I. Mesones, A. Torres-Lezama, L. E. Urrego

425 Giraldo, R. Zagt, M. N. Alexiades, L. Hernandez, I. Huamantupa-Chuquimaco, W. Milliken,

426 W. Palacios Cuenca, D. Pauletto, E. Valderrama Sandoval, L. Valenzuela Gamarra, K. G.

427 Dexter, K. Feeley, G. Lopez-Gonzalez, \& M. R. Silman. 2013. Hyperdominance in the

428 Amazonian tree flora. Science 342, 1243092. (doi:10.1126/science.1243092)

429 ter Steege H., N. C. A. Pitman, T. J. Killeen, W. F. Laurance, C. A. Peres, J. E. Guevara, R. P.

430 Salomão, C. V Castilho, I. L. Amaral, F. Dionízia, D. A. Matos, L. D. S. Coelho, W. E. 
431 Magnusson, O. L. Phillips, D. De Andrade, L. Filho, M. De Jesus, V. Carim, M. V. Irume, M.

432 P. Martins, J. Molino, D. Sabatier, F. Wittmann, D. C. López, J. Renan, A. M. Mendoza, P. N.

433 Vargas, A. G. Manzatto, N. Farias, C. Reis, J. Terborgh, K. R. Casula, J. C. Montero, T. R.

434 Feldpausch, E. N. H. Coronado, A. Javier, D. Montoya, C. E. Zartman, B. Mostacedo, R.

435 Vasquez, R. L. Assis, M. B. Medeiros, M. F. Simon, A. Andrade, J. L. Camargo, S. G. W.

436 Laurance, H. Eduardo, M. Nascimento, B. S. Marimon, B. M. Jr, F. Costa, N. Targhetta, I.

437 Célia, G. Vieira, R. Brienen, H. Castellanos, J. F. Duivenvoorden, H. F. Mogollón, M. Teresa,

438 F. Piedade, G. A. A. C, J. A. Comiskey, G. Damasco, C. Levis, J. Schietti, P. Souza, A.

439 Alonso, F. Dallmeier, L. V. Ferreira, D. Dantas, R. Gribel, B. G. Luize, M. P. Pansonato, E.

440 Venticinque, P. Fine, M. Toledo, C. Baraloto, C. Cerón, J. Engel, T. W. Henkel, E. M.

441 Jimenez, J. Stropp, R. Thomas-caesar, T. R. Baker, D. Daly, M. R. Paredes, N. Ferreira, A.

442 Fuentes, P. M. Jørgensen, J. Schöngart, M. R. Silman, N. C. Arboleda, T. R. Van Andel, P.

443 Von Hildebrand, E. M. Barbosa, L. Carlos, D. M. Bonates, A. A. Oliveira, H. Ramirez-

444 angulo, J. F. Ramos, R. Sierra, M. Tirado, R. Zagt, M. N. Alexiades, K. Garcia-cabrera, L.

445 Hernandez, I. Huamantupa-chuquimaco, W. Milliken, W. P. Cuenca, S. Pansini, D. Pauletto,

446 F. R. Arevalo, A. F. Sampaio, E. H. V. Sandoval, \& L. V. Gamarra. 2015. Estimating the

447 global conservation status of more than 15,000 Amazonian tree species. Science Advances 1,

$448 \quad$ e1500936. (doi: 10.1126/sciadv.1500936)

449 Zanne AE, Lopez-Gonzalez G, Coomes DA, Ilic J, Jansen S, Lewis SL, Miller RB, Swenson

450 NG, Wiemann MC, Chave J. 2009. Data from: Towards a worldwide wood economics

451 spectrum. Dryad Digital Repository. http://dx.doi.org/10.5061/dryad.234

452

453 Data accessibility 
454 DNA sequences: For Genbank accession numbers, see Table S1.

455 Phylogeny: TreeBASE, http://purl.org/phylo/treebase/phylows/study/TB2:S19277?x-access-

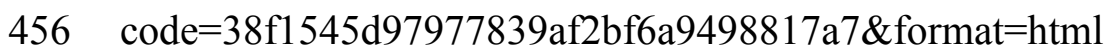

457

458

459

460

461

462

463

464

465

466

467

468

469

470

471

472

473

474

475 
476 Table 1: $\triangle \mathrm{AIC}$ values for different evolutionary models of trait evolution for genus-level

477 characteristics and for different values of Pagel's $\lambda$.

$\begin{array}{llll}\text { Genus characteristic } & \text { Evolutionary Model } & \lambda & \Delta \mathrm{AIC}\end{array}$

\begin{tabular}{lccc}
\hline & estimated $\lambda$ & 0.26 & 0 \\
$\log$ (Species richness) & no phylogenetic dependence & 0 & -17.1 \\
& Brownian motion & 1 & -287.8 \\
& estimated $\lambda$ & 0.37 & 0 \\
$\log$ (Mean range size) & no phylogenetic dependence & 0 & -52.0 \\
& Brownian motion & 1 & -257.9 \\
& estimated $\lambda$ & 0.32 & 0 \\
$\log$ (Mean abundance) & no phylogenetic dependence & 0 & -34.2 \\
& Brownian motion & 1 & -287.5 \\
\hline
\end{tabular}

478

479

480

481

482

483

484

485

486

487 
488 Figure 1: Phylogeny of 631 Amazonian tree genera with terminal branches coloured according

489 to the (A) species richness, (B) mean range size, and (C) mean abundance of genera. The

490 following numbered nodes are mentioned in the main text: 1=Arecaceae, $2=$ Magnoliids,

491 3=Rosids, 4=Asterids, 5=Myrtales, 6=Melastomataceae, 7=Euphorbiaceae, 8=Salicaceae,

492 9=Moraceae, 10=Leguminosae, 11=Lamiales, 12=Rubiaceae, 13=Solanales, and

493 14=Lecythidaceae. Nodes that are coloured blue indicate lineages whose constituent genera show

494 significantly higher values for the given genus-level characteristic than expected by chance,

495 while nodes coloured red show significantly lower values than expected by chance.

496

497 Figure 2: Relationships between species richness and mean range size and abundance for

498 Amazonian tree genera. Histograms of each genus-level characteristic are given on the diagonal.

499 Below the diagonal the raw relationships are shown along with the best-fit linear relationship and

500 the Pearson correlation coefficient. Above the diagonal, the relationships of phylogenetically

501 independent contrasts (PICs) are shown along with the best-fit linear relationship that is forced

502 through the origin and the Pearson correlation coefficient.

503

504

505

506

507

508

509

510 


\section{Figure 1}
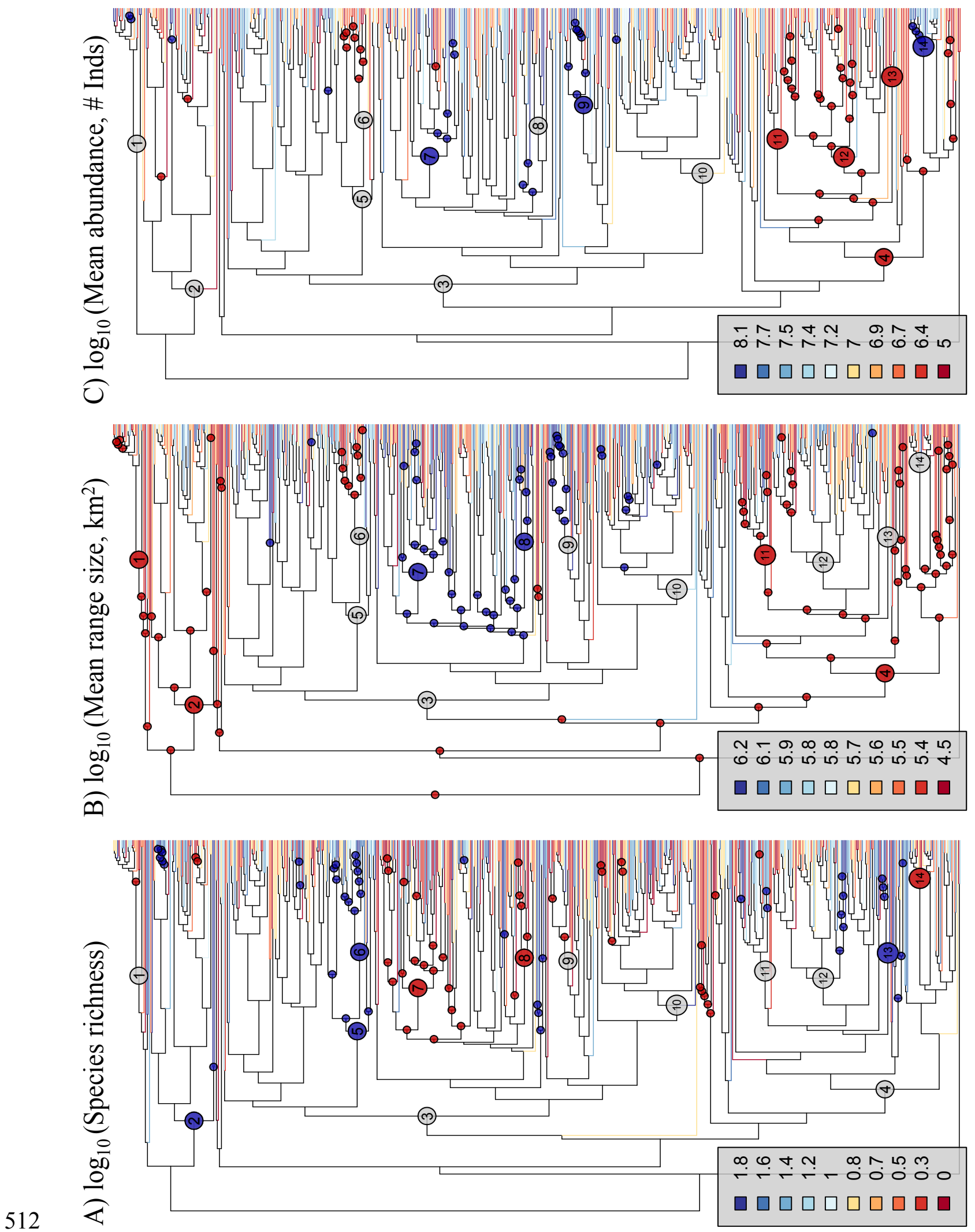


\section{Figure 2}

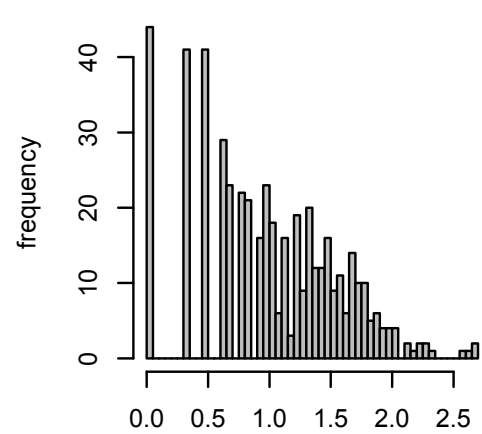

$\log 10$ (species richness)

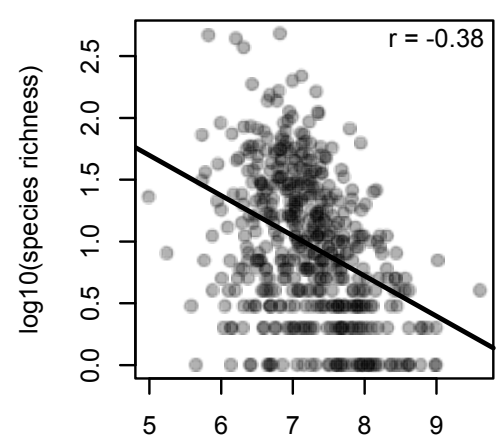

$\log 10$ (mean abunance, \# Inds)

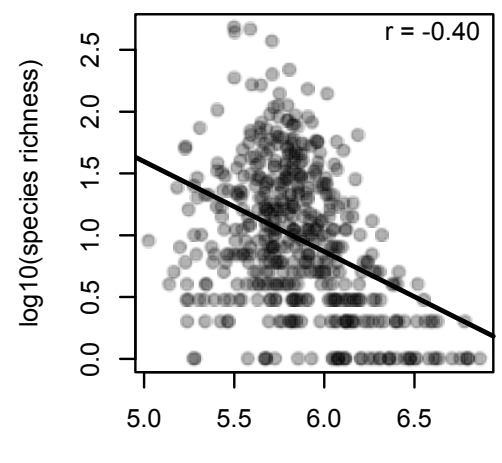

log10(range size, km2)

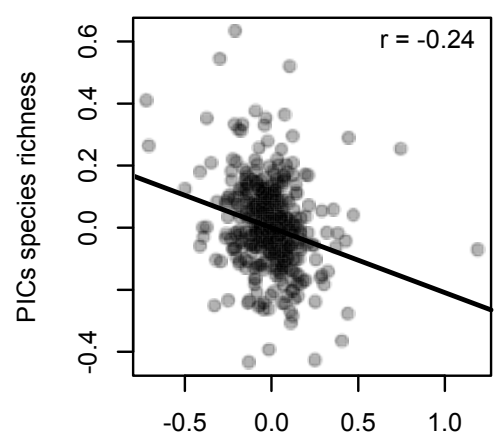

PICs log10(mean abunance, \# inds)

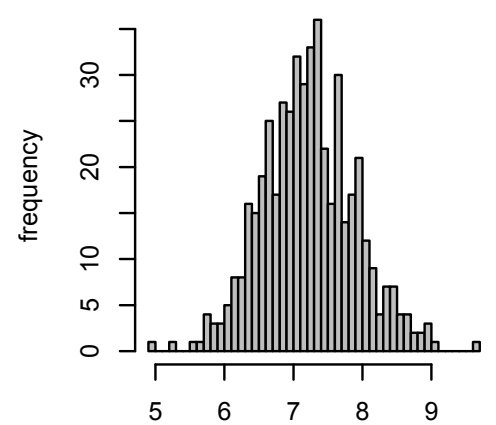

$\log 10$ (mean abunance, \# inds)

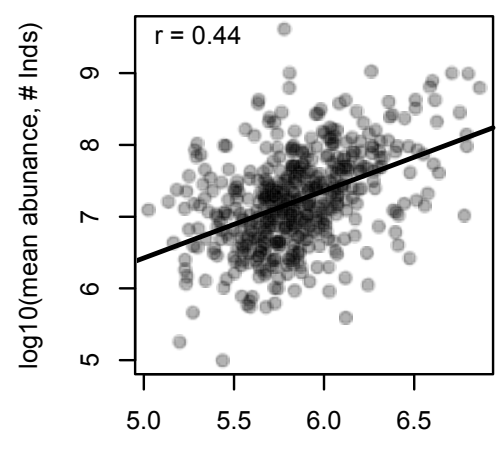

log10(range size, km2)

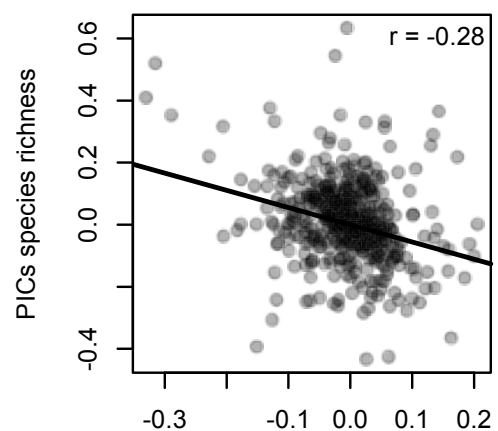

PICs log10(mean range size, km2)

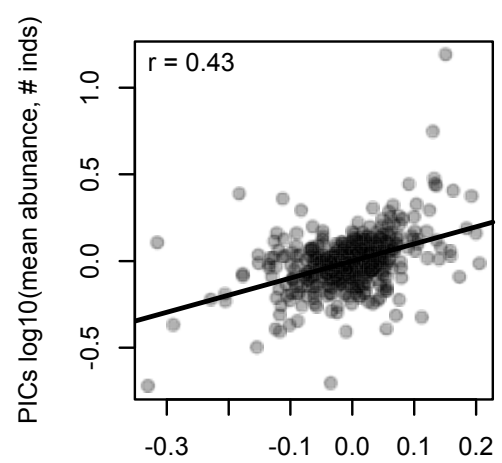

PICs log10(mean range size, km2)

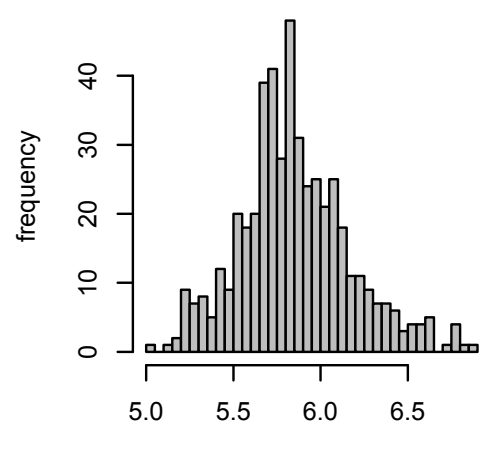

$\log 10$ (mean range size, $\mathrm{km} 2$ ) 


\section{Supplementary Files}

521 Table S1: Amazonian tree genera in the phylogeny generated in this study, along with the

522 species to which each rbcL and matK accession belongs and the associated Genbank accession

523 numbers. For novel sequences, we also give the collection number.

524 Table S2: Nodes in the phylogeny that were constrained based on fossil ages in the temporally-

525 calibrated phylogenetic analysis. The hard minimum age allowed for the node (i.e. the offset)

526 and the mean and standard deviation of the log-normal prior are given, in millions of years.

527 Table S3: Taxonomic lineages present in the phylogeny along with their reconstructed values for

528 species richness, range size and abundance (all log transformed to the base 10 prior to analysis).

529 The probability that a given lineage has a smaller value than that reconstructed is also given,

530 based on a permutation test described in the main text. If this probability is smaller than 0.025 ,

531 then the lineage is described as having a significantly smaller value than expected by chance,

532 while the converse holds if the value is greater than 0.975 .

533 rbcL_matK_alignment.fasta: Final sequence alignment used in phylogenetic analyses. The two

534 genes are concatenated, with rbcL forming the first 1320 base pairs of the alignment.

535 Amazon_genus_phylog_RAxML.tre: Maximum likelihood phylogeny for Amazonian tree 536 genera.

537 Amazon_genus_phylog_BEAST.tre: Temporally-calibrated phylogeny for Amazonian tree 538 genera. This is the all-compatible consensus tree generated from the posterior distribution of 539 trees from a Bayesian phylogenetic analysis, with node ages representing the median value for 540 each node across the posterior distribution of trees.

541 Dexter_AmazonTrees_script.R: The script for all analyses conducted for this manuscript in the 542 R Statistical Software. 
543 taxonomy_table.csv: Infile used in the R script, which contains the taxonomic classification for

544 each genus in the study. 